This is an author produced version of a paper published in 3rd IEEE PES International Conference and Exhibition on Innovative Smart Grid Technologies (ISGT Europe), 2012

This paper has been peer-reviewed but does not include the final publisher proofcorrections or proceedings pagination.

(C) 2012 IEEE. Personal use of this material is permitted. Permission from IEEE must be obtained for all other uses, in any current or future media, including reprinting/republishing this material for advertising or promotional purposes, creating new collective works, for resale or redistribution to servers or lists, or reuse of any copyrighted component of this work in other works.

Citation for the published paper:

Weimer, J., Yuzhe Xu; Fischione, C.; Johansson, K.H.; Ljungberg, P.; Donovan, C.; Sutor, A.; Fahlen, L.E.

A virtual laboratory for micro-grid information and communication infrastructures. 3rd IEEE PES International Conference and Exbibition on Innovative Smart Grid Technologies (ISGT Europe), 2012

Access to the published version may require subscription.

Published with permission from: IEEE 


\title{
A Virtual Laboratory for Micro-Grid Information and Communication Infrastructures
}

\author{
James Weimer, Yuzhe Xu, Carlo Fischione, Karl Henrik Johansson, Per Ljungberg, Craig Donovan, \\ Ariane Sutor, Lennart E. Fahlén
}

\begin{abstract}
Testing smart grid information and communication (ICT) infrastructures is imperative to ensure that they meet industry requirements and standards and do not compromise the grid reliability. Within the micro-grid, this requires identifying and testing ICT infrastructures for communication between distributed energy resources, building, substations, etc. To evaluate various ICT infrastructures for micro-grid deployment, this work introduces the Virtual MicroGrid Laboratory (VMGL) and provides a preliminary analysis of Long-Term Evolution (LTE) as a micro-grid communication infrastructure.
\end{abstract}

\section{INTRODUCTION}

With the recent technological advancements in communication infrastructures, mobile and cloud computing, smart devices, and power electronics, a renewed interest in power systems research has emerged. When these enabling technologies are jointly utilized to sense and actuate power generation, distribution, and demand, the resulting smartgrid has unprecedented capabilities [1]. Some of these capabilities include remotely detecting the statuses of electricity generators, transmission lines and substations; monitoring electricity consumption; adjusting the power consumption of household applications to match supply, and reducing energy losses while increasing electricity grid reliability. As smart-grids begin to emerge, it is imperative to identify and evaluate critical components within the physical, computational, and communication architectures for both commercial product development and societal acceptance.

Within the smart-grid design hierarchy, micro-grids represent localized power grids containing both distribution and generation and arguably have the most to gain from developing new technologies for power conservation, distribution, and localized control. As such, micro-grids present many new challenges from the standpoint of control and communication infrastructures. In response to

J. Weimer, Y. Xu, C. Fischione, and K. Johansson are with the ACCESS research center, School of Electrical Engineering, KTH Royal Institute of Technology, 10044 Stockholm, Sweden. \{jweimer|yuzhe|carlofi|kallej\}@kth.se

P. Ljungberg and C. Donovan are with Ericsson Research, 16480 Stockholm, Sweden. \{per.ljungberg|craig.donovan\}@ericsson.com

L. Fahlén is with the Swedish Institute of Computer Science (SICS), 16429 Kista, Sweden. lef@sics.se

A. Sutor is with Siemens corporate research and is the action line leader of the European Institute of Technology (EIT) Smart Energy Systems (SES) action line, Infopark 1/E - Neumann Janos utca, 1117 Budapest, Hungary. ariane.sutor@siemens.com these challenges, the European Institute of Technology (EIT) Information and Communication Technology (ICT) Labs has introduce the action-line Smart Energy Systems (SES) to develop a Europe-wide coalition of academic and industrial partners and resources in the ICT sector to accelerate innovation in energy management and green ICT management. The virtual micro-grid laboratory described in this work is part of the EIT ICT Labs SES virtual smart grid laboratory activity, where academic and industrial partners from six European countries have joined forces to create a large-scale pan-European smart grid lab.

Within the EIT ICT Labs SES, and motivated by ongoing smart grid pilot research within the Stockholm Royal Seaport project, partners from industry and academia have combined resources to develop a virtual laboratory for testing ICT infrastructures within the micro-grid. Distributed across multiple academic and industrial research labs, this virtual lab provides unprecedented capabilities of evaluating ICT infrastructures for performing energy management related services, such as distribution automation, demand response, and micro-grid control. As a preliminary deployment, the virtual micro-grid lab is designed as a city-level distribution network for evaluating distribution automation and demand-response capabilities ${ }^{1}$ In this work we describe the virtual micro-grid lab architecture, present the first results using the virtual lab, and identify future planned extensions and testing scenarios.

The remainder of this work is organized as follows. The following section describes the high-level hardware, software, and communication architecture of the virtual lab. Section III presents testing requirements and an evaluation of Long-Term Evolution (LTE) as a ICT infrastructure for micro-grids. The concluding section provides a discussion and identifies future work.

\section{Virtual Micro-Grid Lab Architecture}

The virtual lab exists as a collaborative project between academic and industrial partners within the EIT ICT Labs to investigate ICT infrastructures for micro-grid communication and control. Each participant has employed their respective expertise to develop specific micro-grid components and functionality, as illustrated in Fig. 1. The

\footnotetext{
${ }^{1}$ While the virtual lab, in its current deployment, does not contain distributed generation capabilities and would more accurately be classified as a city-level distribution network, inclusion of distributed generation and storage into the virtual lab are underway. Thus, for purposes of naming continuity in future publications, we refer to the virtual lab as a micro-grid.
} 


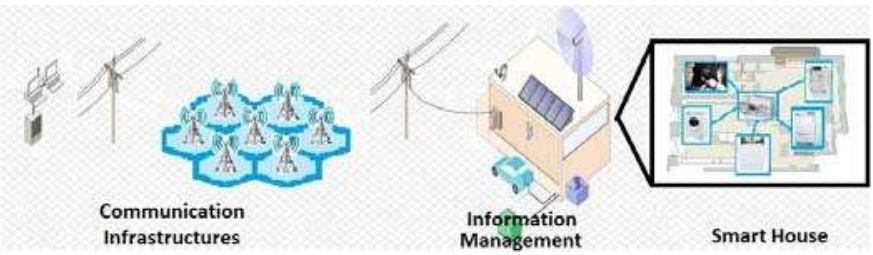

Fig. 1. Virtual Laboratory Architecture.

physical architecture is such that each academic and industrial member participating in the laboratory can exploit local expertise to develop micro-grid functionalities. Then as a collaboration, these individual components are evaluated over various communication networks for the purpose of hardware-in-the-loop evaluation of the communication infrastructure. As depicted by Fig. 1, the virtual microgrid lab architecture consists of three main components, (a) micro-grid communication infrastructures for microgrid applications such as inter-substation communication, substation-to-building communication, and distributed energy resources, (b) micro-grid information management systems for distribution automation, demand-side scheduling, distributed generation/control, and the smart home energy management system (EMS), (c) communication technologies and incorporation of smart appliances within the smart home EMS. The following subsections respectively discuss the Smart House, Information Management, and Communication Infrastructures utilized within the virtual lab in detail.

\section{A. Smart House}

The smart house represents the lowest-level component of the virtual lab and consists of all the physical devices which exist inside a smart home. The devices containing communication capabilities consist of the residential gateway, smart appliances, and the home automation system. Specifically, the smart home interfaces to the microgrid via a smart home EMS which coordinates with the home automation system and smart appliances through a residential gateway. The remainder of this subsection describes each component of the smart house in detail.

The residential gateway serves as a platform for hosting the smart home EMS and communicates with the home automation system, smart appliances, and the micro-grid. Within the virtual lab, the residential gateway is based on a Java open service gateway initiative (OSGi) framework and contains the physical WAN (wide area network) and HAN (home area network) communication enablers. Different communication stacks and protocol stacks for smart home appliances and home automation profiles using e.g. ZigBee, ZWave, CoAP, and KNX (via an IP Gateway) to be used in different virtual lab configurations.

Smart appliances are an integral part of the virtual laboratory and represent appliances which are either completely or partially controlled by the smart house Energy Management Systgem - EMS. In the virtual lab experiments, the smart appliances communicate with the smart house EMS via a ZigBee home automation profile adaptor in the OSGi residential gateway.

A micro-grid could consists of a suburb having 10,000 smart houses. While each smart house has local control and scheduling capabilities for smart appliances and the home automation system, to ensure micro-grid stability and functionality requires the global management of pertinent information such as current generation and demand profiles. The coordination of this micro-grid information is handled by the information management systems as described in the following subsection.

\section{B. Information Management}

In order to meet the constraints on demand and distributed generation, information must be shared between the end-user smart-homes. Within the micro-grid, there are three prominent information management systems, namely the demand response management system (DRMS), the smart house energy management system (EMS), and the end-user home control application. The remainder of this subsection describes the DRMS, EMS, and the home control application in detail.

The DRMS is responsible for generating demand response messages, at the micro-grid level, based on the current electrical load of the power grid and the anticipated future demand. The purpose of the DRMS in generating demand response messages is to promote grid stability and environmental responsibility by offering incentives to endusers for adjusting their power usage by providing future electric pricing and $\mathrm{CO}_{2}$ emission figures. Additionally, the demand response message may contain an explicit load-reduction request based on contractual agreements between the electric provider and end-users. The demand response messages are transmitted via a communication network to the EMS local to each end-user.

The smart house EMS is responsible for locally scheduling end-user smart appliances at the residential level. The EMS interprets the messages generated by the microgrid demand response management system (DRMS) and schedules the smart devices based on end-user preferences, prior contractual agreements, and knowledge of controllable smart devices. Within the virtual micro-grid lab, the EMS is implemented in Java as an OSGi bundle and is operated on the residential gateway.

The end-user home control application is a mobile web application that enables the user to remotely change user preferences on the EMS. While the primary purpose from an information management point-of-view is to provide functionality for changing the operating preferences of the smart house EMS, the control application is also capable of visualizing energy usage data within the home to provide real-time feedback of energy usage and carbon dioxide generation.

All of the information management systems within the micro-grid must communicate in order to meet constraints on power usage and carbon dioxide emissions. Identifying satisfactory and economically feasible communication infrastructures for various micro-grid functionalities (such 
as demand response, distribution automation, and distributed generation and control) is a primary focus of the virtual lab. The following subsection describes the potential communication infrastructures to be considered.

\section{Communication Infrastructures}

The primary purpose of the virtual micro-grid lab is to study communication infrastructures for various microgrid functionalities. Within the virtual lab, multiple communication platforms are anticipated to be utilized for various applications. These communication infrastructures include, but are not limited to, fixed broadband, LTE/4G, and machine-to-machine communication protocols.

Fixed broadband communication consists of hard-wired networks, such as fiber optics, which represent the fastest communication medium available within the micro-grid. While their data rates can be significantly faster and more reliable than other wireless mediums, the installation and service costs associated with a micro-grid fixed broadband network limits its implementation. In future microgrid communication networks, it is anticipated that fixed broadband networks will be utilized when alternative networks can not meet industry standards and requirements.

3GPP Long Term Evolution (LTE), the latest wireless communication technology, is a promising option for the smart grid [2], [3]. LTE was developed to fulfil mobile users' demands for higher data rates and stabler service performance. An attractive feature of the LTE network is that existing telecommunication operator networks could be utilized, thus reducing the overall cost of implementation. However, LTE has not been designed specifically for smart grid applications and therefore its suitability is currently being studied within the virtual micro-grid lab. Utilizing the LTE network for micro-grid communication will be addressed in detail in the following section.

The M2M platform offers the applications a uniform addressing of resources and a single-point of integration. It handles secure messaging of demand response messages from the application to the residential gateway and gives the end-user home control application access to sensors and actuators.

In this subsection we have introduced a virtual microgrid laboratory for evaluating information and communication infrastructures. The architecture of the virtual lab, from both the physical components and the intercomponent communication, is described. The virtual laboratory described in this section is employed in the following section to provide an initial assessment of Long Term Evolution (LTE) for micro-grid communication.

\section{LTE FOR Micro-Grid Communication}

The 3GPP white paper and LTE service operators announced that the main advantages with LTE are to provide high throughput (up to $300 \mathrm{Mbps}$ in downlink, and 75 Mbps in uplink), low latency (less than $100 \mathrm{~ms}$ for control plane latency, and less than $5 \mathrm{~ms}$ for user plane latency), self-organizing networking capabilities (plug and play), Frequency (FDD) and Time Division Duplex (TDD) in the same platform, an improved end-user experience and a simple all IP architecture resulting in low operating costs [2]. The measurement requirements for smart grid are defined or introduced in technical literature. Standards [4]-[6] introduce the concepts such as message types and reporting rates for electricity substations, Phasor Measurement Units, and automatic meter readings. The topologies and communication infrastructures of a smart grid are discussed in [7]-[12]. Literatures [13]-[15] introduce several LTE scheduler designs for common user equipment as mobile phones, which have quite different requirements compared to smart grids components. A preliminary assesment of LTE for smart grid applications has been performed in [16], but this work does not attempt to quantify a latency distribution. The availability of a latency distribution is essential to design controllers that are able to take real-time actions on the smart grids. It is also essential that such a distribution meets the specific requirements of smart grids components.

In the remainder of this section, preliminary experiments are described and conducted to evaluate the roundtrip latency of communication via LTE.

\section{A. Latency Requirement for Communication in Smart Grid}

For the smart grid communication network, latency is one critical technical requirement. Measurements and commands must be available within specific delays based on the application area. Other researchers have considered micro-grid latency requirements [17]-[19]. To summarize the latency requirements, various smart grid components require different latencies ranging from less than $3 \mathrm{~ms}$ for protection commands within the substation to between 20 and $100 \mathrm{~ms}$ for distribution automation commands in normal operating modes (non-transient).

In most cases, a smart grid focuses on three main areas: household devices and automatic meter reading; remote sensing devices for grid monitoring and control, and distributed energy resource, such as wind and solar management. The key components in a smart grid are: the Advanced Meter Infrastructures (AMI) at houses or buildings, Phasor Measure Units (PMU) for transmission lines and power generations such as distributed generations and substations. In the following, we describe these components in detail with focus on their latency requirements.

PMUs provide phasor measurements of voltages and currents in an electrical grid for high fidelity sensing. The phasor measurements are calculated via Discrete Fourier Transform (DFT) and delivered to devices called Phasor Data Concentrators (PDC). In PDC, the measurements are time-synchronised, stored for future reference and forwarded to application and Super PDCs. Generally speaking, PMU measurements are 100 200 bytes and reported at a rate of about 4000 times a second. They are expected to meet real-time control system requirements with time delay less than $10 \mathrm{~ms}$ [17]. 
AMI is an upgrade of Automatic Meter Reading (AMR) providing two-way communication and specific actuators. AMI collects information of consumption records, alarms and status from customers and can impose consumption control. Based on its two-way communication and consumption metering, AMI enables real time pricing and peak shaving in a smart grid. Referring to Wide-Area Measurement System (WAMS), latency less than 1 second (typically 100 200 ms) is required to achieve real time pricing requirements [18], [19].

The communication requirements for functions and device models in a substation are defined by Standard IEC 61850-5 [4]. The size of message varies from 1 to 1024 bits. Those messages delivery latencies vary from $3 \mathrm{~ms}$ to $1 \mathrm{~s}$. However, to be viable for distribution automation requires that the LTE network latency is less than $10 \mathrm{~ms}$ for interconnecting PMUs and AMIs. In the following subsection, we investigate the LTE latency for LTE communication.

\section{B. Experimental Setup}

In this subsection, we describe the experimental setup for empirically evaluating the latency offered by LTE. Experiments were performed using off-the-shelf LTE USB modems. The experimental results presented in this subsection are analyzed in the following subsection. To evaluate the round-trip-times (RTT) for transmission and reception, Internet Control Message Protocol (ICMP) echo request packets are sent to the target host via the LTE modem, and the ICMP response time recorded. The round trip time (RTT) values for transmission and reception were then analyzed.

We considered two LTE network services in Stockholm, Sweden, managed by separate Operators. The RTT and data loss rate of messages traveling between client and service are measured and recorded. The length of messages varies from 0 bytes to 1024 bytes. Every message corresponds to one or a group of readings generated by the components of the smart grid.

\section{Experimental Results}

Fig. 2 shows the mean values and standard deviations of the RTT measured by ping command using for LTE networks. Fig. 2(a) illustrates the values for small data packets, less or equal to 100 bytes, while Fig. 2(b) shows those for larger packets, up to 1000 bytes.

These figures indicate that when the length of data packets is smaller than 100 bytes, the RTT is shorter than 20 ms under the service provided by Operator 1 , while it is around $20 \mathrm{~ms}$ for Operator 2. The RTT values increase with the length of data packets when the length is larger than 100 bytes. But the standard deviations of RTT are approximately the same whatever sizes for the packets for each Service Operator. The mean values of RTT under Operator 1 are lower than those under Operator 2. However, the standard deviation of RTT under Operator 1 is around 4 5 times larger than that under Operator 2. In

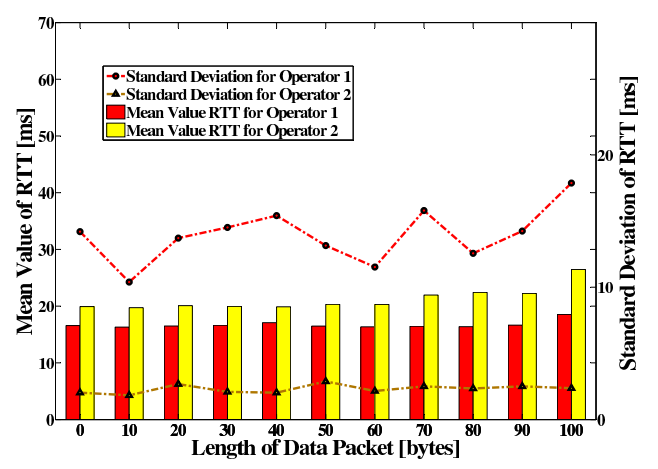

(a) Small data packets

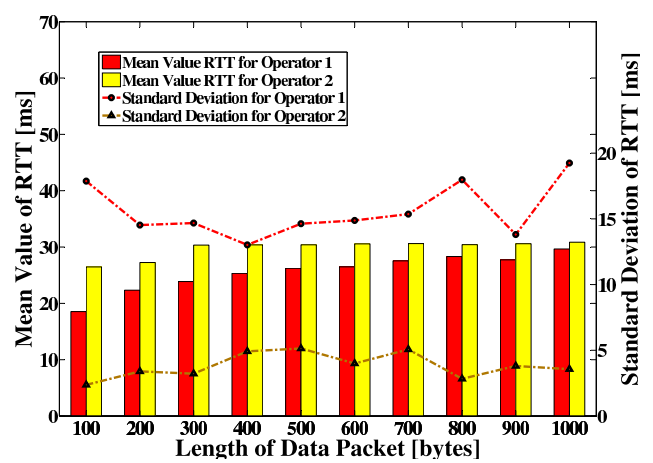

(b) Large data packets

Fig. 2. Mean values and standard deviations of RTT

addition, the minimum latencies for RTT transmission are $10 \mathrm{~ms}$ and $13 \mathrm{~ms}$ via Operation 1 and 2 respectively.

By dividing RTT in half to calculate the latency, the minimum values of the latency for small size packet agrees with the theoretical latency given by 3GPP white paper, which is $5 \mathrm{~ms}$. However, note from Fig. 2(a) that this has a low probability of occurrence.

1) Latency Distribution Model: An accurate latency distribution model is necessary to predict the communication latency via LTE network. We propose to model the probability density function (pdf) for RTT as

$$
\begin{aligned}
f(x)= & \sum_{i=0}^{H} \sum_{j=0}^{H}\left(1-p_{\text {up }}\right)\left(1-p_{\text {down }}\right) p_{\text {up }}^{i-1} p_{\text {down }}^{j-1} \\
& N\left(\mu-i T_{\text {up }}-j T_{\text {down }}, \sqrt{\sigma_{\text {up }}^{2}+\sigma_{\text {down }}^{2}}\right),
\end{aligned}
$$

where $T_{\mathrm{HARQ}}$ is the time spent to re-send the data packet across $H$ maximum times of HARQ retransmissions, $p_{\text {up }}$ and $p_{\text {down }}$ are the probabilities of repeating request, $T_{\text {up }}$ and $T_{\text {down }}$ are the time spent on resending in up- and down-link respectively, and $N\left(\mu-i T_{\text {up }}-\right.$ $\left.j T_{\text {down }}, \sqrt{\sigma_{\text {up }}^{2}+\sigma_{\text {down }}^{2}}\right)$ is pdf for normal distribution. Using maximum likelihood (MLE) and least squares estimation (LSE), the parameters that give the "best" fitting distribution functions can be obtained. The estimated values for those parameters in the model are listed in Tab. I, and the fitting results are shown in Fig. 3. According to the results, one of the estimated time for $T_{\mathrm{HARQ}}$ is approximately $8 \mathrm{~ms}$. 
Table I Summary of fits of RTT values for both Operators in Fig. 3

\begin{tabular}{llcccc}
\hline & & \multicolumn{2}{c}{ Operator 1 } & \multicolumn{2}{c}{ Operator 1 } \\
& & MLE & LSE & MLE & LSE \\
\hline SSE & $r^{2}$ & 0.81 & 0.82 & 0.99 & 1.00 \\
Mean Value [ms] & $\mu$ & 13.92 & 14.18 & 26.32 & 26.37 \\
Standard Deviation & $\sigma$ & 1.71 & 1.79 & 2.02 & 1.85 \\
HARQ Time [ms] & $T_{\text {up }}$ & 7.85 & 7.51 & 8.72 & 9.27 \\
& $T_{\text {down }}$ & 1.00 & 0.00 & 1.09 & 0.50 \\
HARQ Probability & $p_{\text {up }}$ & 0.19 & 0.22 & 0.02 & 0.02 \\
Max. Resend Times & $p_{\text {down }}$ & 0.00 & 0.01 & 0.00 & 0.15 \\
\hline
\end{tabular}

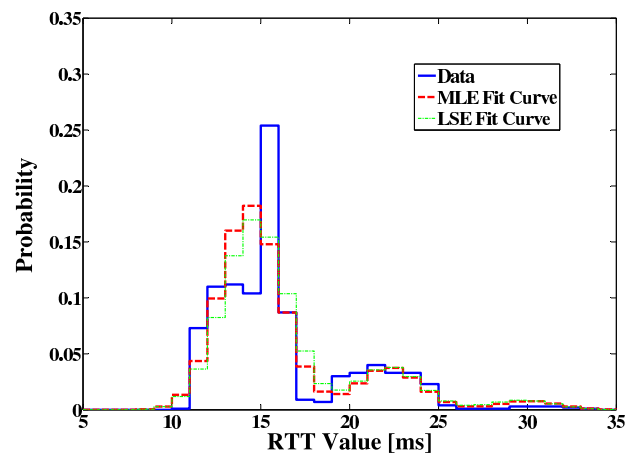

(a) Via Operator 1 LTE network

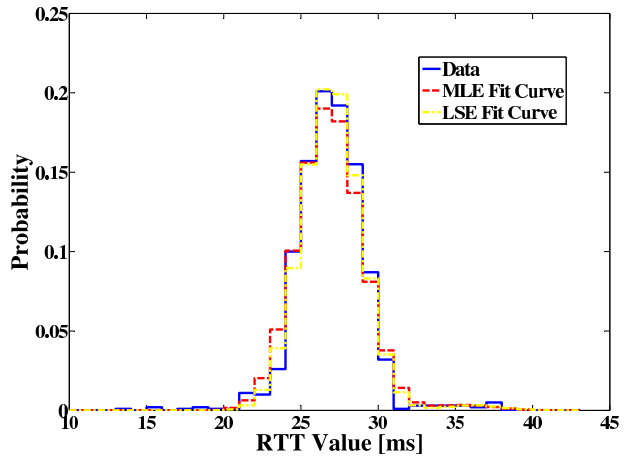

(b) Via Operator 2 LTE network

Fig. 3. RTT values distribution. The data set is collected via LTE network with 100 bytes data packets

From the experimental results it follows that the minimum latencies for less than 300 bytes data packets are close to the theoretical latency announced by the 3GPP white paper (less than $5 \mathrm{~ms}$ one-way). However, most of the packets are transmitted with a latency that is around $15 \mathrm{~ms}$ for Operator 1 and $25 \mathrm{~ms}$ for Operator 2. These results indicate that if smart grid components require latencies of less than $15 \mathrm{~ms}$, then the LTE network must be optimized. This can be achieved through developing new scheduler algorithms for the LTE radio base station such that the latency is reduced.

\section{Conclusions}

In this work, a virtual micro-grid laboratory for testing ICT infrastructures is introduced. The lab architecture is described and a preliminary practical evaluation of LTE for smart grid communication is presented. Based on this successful evaluation, future testing scenarios related to demand-response, distribution automation, and microgrid control are planned, as well as extensions to include capabilities to both emulate ICT infrastructures within the micro-grid and to consider distributed generation scenarios. Experiments are currently planned for evaluating demand response within a smart grid as well as microgrid control. The virtual micro-grid lab is expected to be utilized by the Stockholm Royal Seaport project to identify useful communication infrastructures for microgrid operations.

\section{REFERENCES}

[1] H. Farhangi, "The path of the smart grid," IEEE Power and Energy Magazine, vol. 8, pp. 18-28, Jan. 2012.

[2] S. Sesia, I. Toufik, and M. Baker, LTE: The UMTS Long Term Evolution from theory to practice, John Wiley \& Sons Ltd., 2009.

[3] P. Lescuyer and T. Lucidarme, Evolved Packet System (EPS): The LTE and the SAE Evolution of $3 G$ UMTS. John Wiley \& Sons Ltd., 2008.

[4] Communication Requirements for Functions and Device Models, IEC Standard 61850-5, 2003.

[5] IEEE Standard for Synchrophasors for Power Systems, IEEE Standard C37.118, March 2006.

[6] Electricity Metering Data Exchange for Meter Reading, Tariff and Load Control: Part 21 Direct Local Data Exchange, IEC Standard 62056-21, 2002.

[7] V.K. Sood, D. Fischer, J.M. Eklund, and T. Brown, "Developing a communication infrastructure for the smart grid," in IEEE Electrical Power and Energy Conference (EPEC), 2009, pp. 17 .

[8] D.E. Bakken, A. Bose, C.H. Hauser, D.E. Whitehead, and G.C. Zweigle, "Smart generation and transmission with coherent, real-time data," Proceedings of the IEEE, vol. 99, pp. 928-951, Jun. 2011.

[9] Y.J. Kim, M. Thottan, V. Kolesnikov, and W. Lee, "A secure decentralized data-centric information infrastructure for smart grid," IEEE Communication Magazine, vol. 48, pp. 58-65, Nov. 2010.

[10] K.C. Sou, J. Weimer, H. Sandberg, and K.H. Johansson, "Scheduling smart home appliances using mixed integer linear programming," In IEEE Conference on Decision and Control (CDC) 2011, pp. 5144-5149.

[11] D. Zhang, L.G. Papageorgiou, N.J. Samsatli, and N. Shah, "Optimal scheduling of smart homes energy consumption with microgrid," In ENERGY 2011, pp. 70-75.

[12] A. Aggarwal, S. Kunta, and P.K. Verma, "A proposed communication infrastructure for the smart grid," In Innovative Smart Grid Technologies (ISGT), 2010, pp. 1-5.

[13] H.K. Yang, F.Y. Ren, C. Lin, and J. Zhang, "Frequency-domain packet scheduling for 3gpp lte uplink," In IEEE INFOCOM 2010, pages 1-9.

[14] S.B. Lee, S. Choudhury, and A. khoshnevis, "Downlink mimo with frequency-domain packet scheduling for 3gpp lte," In IEEE INFOCOM 2009, pp. 1269-1277.

[15] S. Lee, I. Pefkianakis, and A. Meyerson, "Proportional fair frequency-domain packet scheduling for 3gpp lte uplink," In IEEE INFOCOM 2009, pp. 2611-2615.

[16] B. Zhen P. Cheng, L. Wang and S. Wang, "Feasibility study of applying lte to smart grid," In First International Workshop on Smart Grid Modeling and Simulation (SGMS), SmartGridComm 2011, pp. 108-113.

[17] C.H. Hauser and D.E. Bakken, "Security, trust, and qos in nextgeneration control and communication for large power system," International Journal of Critial Infrastructures, 2007.

[18] J.Y. Cai, Z. Huang, J. Hauer, and K. Martin, "Current status and experience of wams implementation in north america," In IEEE/PES Transmission and Distribution Conference and Exhibition (Asia and Pacific), 2005, pp 1-7.

[19] T. Khalifa, K. Naik, and A. Nayak, "A survey of communication protocols for automatic meter reading applications," IEEE Communication Survey and Tutorials, vol. 13, pp. 168-182, Second Quarter 2011. 


\section{BIOGRAPHIES}

James Weimer received his B.S. in Electrical Engineering from Purdue University in 2005 and his M.S. and Ph.D. degrees in Electrical and Computer Engineering from Carnegie Mellon University in 2007 and 2010, respectively. He is currently a post-doc researcher in the Automatic Control Lab at the KTH Royal Institute of Technology in Stockholm, Sweden where he specializes in security and control of networked systems with an emphasis on implementable applicationoriented solutions. He is an active participant within the European Institute of Technology (EIT) and is currently the task co-leader for the Virtual Micro-Grid Lab within the Smart Energy Systems action line.

Yuzhe $\mathrm{Xu}$ received the B.E. degree in electrical engineering from Shanghai Jiao Tong University, China, in 2005, and M.E. degree in electrical engineering from KTH Royal Institute of Technology, Sweden, in 2011. He is currently pursuing the Ph. D. degree in electrical engineering at KTH Royal Institute of Technology. His current research interests include smart grids, distributed optimization, and wireless sensor networks.

Carlo Fischione is a tenured Associate Professor at KTH Royal Institute of Technology, Electrical Engineering and ACCESS Linnaeus Center, Automatic Control Lab, Stockholm, Sweden. He received the Ph.D. degree in Electrical and Information Engineering in May 2005 from University of L'Aquila, Italy, and the Dr.Eng. degree in Electronic Engineering (Laurea, Summa cum Laude, $5 / 5$ years) in April 2001 from the same University. He held research positions at University of California at Berkeley, Berkeley, CA (2004-2005, Visiting Scholar, and 2007-2008, Research Associate) and Royal Institute of Technology, Stockholm, Sweden (2005-2007, Research Associate). His research interests include optimization and parallel computation with applications to wireless sensor networks, networked control systems, and wireless networks. He has co-authored over 70 publications in international journals and conferences and an international patent. He received numerous awards, including the best paper award from the IEEE Transactions on Industrial Informatics of 2007, the best paper awards at the IEEE International Conference on Mobile Adhoc and Sensor System 05 and 09 (IEEE MASS 2005 and IEEE MASS 2009), the Best Business Idea award from VentureCupEast Sweden, 2010, the "Ferdinando Filauro" award from University of L'Aquila, Italy, 2003, the "Higher Education" award from Abruzzo Region Government, Italy, 2004, and the Junior Research award from Swedish Research Council, 2007, the "Silver Ear of Wheat" award in history from the Municipality of Tornimparte, Italy, 2012. He has chaired or served as a technical member of program committees of several international conferences and is serving as referee for technical journals. Meanwhile, he also has offered his advice as a consultant to numerous technology companies such as Berkeley Wireless Sensor Network Lab, Ericsson Research, Synopsys, and United Technology Research Center. He is Member of IEEE (the Institute of Electrical and Electronic Engineers), SIAM (the Society of Industrial and Applied Mathematics), and Ordinary Member of DASP (the academy of history Deputazione Abruzzese di Storia Patria).

Craig Donovan received the Bachelor of Surveying (BSurv) degree from Otago University, New Zealand in 1994. He is a Registered Surveyor and has worked for over a decade installing both power and fiber optic submarine cables. In 2009 he received the M.Sc in Environmental Engineering from the Royal Institute of Technology (KTH), Stockholm Sweden. He is currently a Senior Researcher at Ericsson Research in Stockholm. His research interests include sustainability assessment and technical evaluation of smart grid communication solutions, with a particular focus on Long Term Evolution (LTE) wireless technology for distribution automation. He is also the principle driver for Ericsson's partnership in InnoEnergy within the European Institute of Technology (EIT).
Karl Henrik Johansson is Director of the KTH ACCESS Linnaeus Centre and Professor at the School of Electrical Engineering, Royal Institute of Technology, Sweden. He is a Wallenberg Scholar and has held a Senior Researcher Position with the Swedish Research Council. He received MSc and $\mathrm{PhD}$ degrees in Electrical Engineering from Lund University. He has held visiting positions at UC Berkeley (1998-2000) and California Institute of Technology (2006-2007). His research interests are in networked control systems, hybrid and embedded control, and control applications in automotive, automation and communication systems. He was a member of the IEEE Control Systems Society Board of Governors 2009 and the Chair of the IFAC Technical Committee on Networked Systems 2008-2011. He has been on the Editorial Boards of Automatica (2003-2006) and IEEE Transactions on Automatic Control (2008-2010), and is currently on the Editorial Boards of IET Control Theory and Applications and the International Journal of Robust and Nonlinear Control. He was the General Chair of the ACM/IEEE Cyber-Physical Systems Week (CPSWeek) 2010 in Stockholm. He has served on the Executive Committees of several European research projects in the area of networked embedded systems. In 2009, he received the Best Paper Award of the IEEE International Conference on Mobile Ad-hoc and Sensor Systems. He was awarded an Individual Grant for the Advancement of Research Leaders from the Swedish Foundation for Strategic Research in 2005. He received the triennial Young Author Prize from IFAC in 1996 and the Peccei Award from the International Institute of System Analysis, Austria, in 1993. He received Young Researcher Awards from Scania in 1996 and from Ericsson in 1998 and 1999.

Per Ljungberg is Research Program Manager at Services and Software, Ericsson Research Stockholm, Sweden. His current research focus is on ICT solutions for Smart Grids, representing Ericsson in European Institute of Technology ICT - Smart Energy Systems action line. He joined Ericsson in 1990 and has since worked with software development and systems architecture for mobile systems (GSM/UMTS) in numerous R\&D positions in Stockholm and at Ericsson Eurolab in Aachen, Germany. Within Ericsson, he was the manager, system engineering at Business Unit Multimedia from 20042008 and since 2008 he has been with the business development and applied research for industry specific solutions. Per holds a Master's Degree in Computer Science from the University of Linköping, Sweden.

Ariane Sutor is the action line leader of smart energy systems at EIT ICT Labs as of September 2011. She thereby coordinates activities of more than 30 leading industrial and academic partners in 7 European countries. Ariane Sutor has been working for Siemens Corporate Technology since 2004. Previously, she held positions as a program manager for IT Security and for Reliability Availability. She received an MSc in mathematics and business management from TU Munich and a PhD from Universität der Bundeswehr, where she is also a lecturer.

Lennart E. Fahlén is the lab manager of the Interactive Collaborative environments Laboratory, ICE, at SICS, which researches middleware platforms for event distribution and interaction over hybrid networks (mobile terminals, micro-controllers etc.) and tools and techniques for distributed virtual environments, IoT sensor applications and novel interaction and social media artifacts. He has an interest in the application of networked distributed ubiquitous computing techniques to the area of energy consumption awareness and control with a special view towards the future development of electric cars and related infrastructures as well as trying to bring a competitive advantage to the European theatre of commerce. Mr Fahlén was for two years a member of the Swedish National Defense's Networked-Based Defense Advisory Board and has held a patent in the area of computer architecture as well as participating in the EU projects COMIC, COVEN, ESCAPE, KIDSTORY, PING, ACCORD, IPERG, ME3GAS and the national energy-awareness projects ERG, FOE and HYRBRIDSPACES. 